\title{
Uncertain Network Public Sentiment Emergency Decision Model Based on Grey Relational Degree
}

\author{
Qiansheng Zhang ${ }^{1, *}$, Xinmeng Zhang ${ }^{2}$ and Bailin Xie ${ }^{2}$ \\ ${ }^{1}$ School of Finance, Guangdong University of Foreign Studies, Guangzhou 510006, China; ${ }^{2}$ School of Informatics, \\ Guangdong University of Foreign Studies, Guangzhou 510006, China
}

\begin{abstract}
This paper presents a novel warning decision model for uncertain network public sentiment emergency of which index assessments are provided as uncertain values. First, a weighted grey relational formula is proposed to compute the comprehensive grey relational degree between the unknown network public sentiment emergency and each original emergency case. According to the evaluated grey relation degree of all the candidate cases, we can easily rank all the matched cases and get the most optimal source case. Also the related emergency section may use or modify the corresponding decision solution of the most matched historical case to facilitate the urgent decisionmaking of current network public sentiment emergency with incomplete information.
\end{abstract}

Keywords: Case matching, distance, emergency decision model, grey relational degree, warning decision index.

\section{INTRODUCTION}

Network public sentiment emergency early warning and Decision making is a very imperative problem in the government crisis management field. In fact, network public sentiment emergency may be induced by many uncertain risk factors. Additionally, in the short time after some network sentiment emergency occurring, the related emergency decision-makers usually collect incomplete and uncertain emergency warning decision index values such as interval value, fuzzy value and vague value.

Recently, a number of emergency management methods and decision models for public emergencies have been designed [1-3]. Also, many researchers proposed some warning decision indexes for network public sentiment emergency [4, 5] and give some warning decision mechanism and strategies [6]. Later, network public sentiment emergency early warning models have been studied by fuzzy theory, statistical method and neural network. For example, Zeng [7] employed the fuzzy hierarch analysis method to determine the weight of early warning index and then proposed some related warning decision model. Lin [8] and Dai [9] utilized fuzzy reasoning rule and fuzzy comprehensive evaluation method to study the network sentiment emergency warning decision model. Li [10] also presented the grey warning decision method for network sentiment emergency. Xie [11] illustrated the decision strategies in different life cycles of network sentiment emergency. Recently, by employing the web text mining technology, some computer aided network sentiment emergency supervision decision system model have been designed [12-14]. Recently, Zhong [15] presented the case-based warning decision model for emergency system. The above-mentioned early warning decision system

*Address correspondence to this author at the Baiyun Road North No. 2, Guangzhou 510420, China; Tel: 020-26270362;

E-mail: zhqiansh01@126.com model can avoid the limitation of traditional risk earlywarning analysis, most of the existing case-based reasoning models of emergency warning decision system utilize similarity measure of the unexpected emergency to the historical cases in emergency case base. And most related literature use the nearest neighbor algorithm to retrieve the optimal early warning decision case and further facilitate emergency decision case. However, the above-mentioned network sentiment emergency warning decision system models are not very efficient to evaluate the comprehensive risk warning degree and measure the similarity between fuzzy warning decision indexes of network sentiment emergency, so they can not make efficient urgent decision for uncertain network sentiment emergency involved with multiple fuzzy indexes.

As is well known, the case-based reasoning efficiency of network sentiment public emergency greatly depend on the used retrieval algorithm. In order to overcome the shortcoming of using nearest neighbor algorithm in case reasoning process, in this paper we aim to develop an improved casebased reasoning method for uncertain network public sentiment emergency by using grey relational degree $[16,17]$ to replace similarity. Also, a new early warning decision model for uncertain network public sentiment emergency based on grey relational retrieval algorithm is then presented. By computing the comprehensive grey relational degree between the current network sentiment emergency and each stored emergency cases in the designed warning decision system, we can easily retrieve the most nearest historical case and then facilitate the warning decision of the current unknown network sentiment emergency based on the resolution of the optimal network sentiment emergency source case.

\section{PRELIMINARIES}

Due to the increasing complexity of real-life socioeconomic environment and the lack of knowledge about the 
problem domain, in the process of uncertain network public sentiment emergency warning decision, emergency decision makers are often provided with some incomplete and uncertain index value of network sentiment emergency. Generally, each network sentiment emergency possibly contains multiple fuzzy warning decision indexes [18].

In order to compare the similarity between two network sentiment emergencies with fuzzy indexes and further match the warning decision resolution of current unexpected emergency problem, we need to propose the following basic notions.

Assume there are two network public sentiment emergencies $y$ and $x$ with $\mathrm{n}$ warning decision indexes. Suppose $d$ is the distance between the jth common index value of the two network sentiment emergencies. Due to the different type of warning decision index of network public sentiment emergency, in this paper we shall employ the different distance measure between index values as below.

(1) If the jth index value of network sentiment emergency is

crisp data, then the distance between them can be evaluated by

$$
d\left(y_{j}, x_{j}\right)=\left|y_{j}-x_{j}\right|,
$$

where $\max _{j}, \min _{j}$ are the maximum and minimum value of jth index of emergency.

(2) If the jth index value of network sentiment emergency are interval number as $y_{j}=\left[y_{j 1}, y_{j 2}\right], x_{j}=\left[x_{j 1}, x_{j 2}\right]$, then the distance between them is evaluated by

$d\left(y_{j}, x_{j}\right)=\sqrt{\left[\left(y_{j 1}-x_{j 1}\right)^{2}+\left(y_{j 2}-x_{j 2}\right)^{2}\right] / 2}$

(3) If the jth index value of network sentiment emergency are intuitionistic fuzzy numbers $[19,20]$ as $y_{j}=<\mu_{y_{j}}, v_{y_{j}}>$, $x_{j}=<\mu_{x_{j}}, v_{x_{j}}>$, then the distance between them is given by $d\left(y_{j}, x_{j}\right)=\max \left\{\left|\mu_{y_{j}}-\mu_{x_{j}}\right|,\left|v_{y_{j}}-v_{x_{j}}\right|\right.$

(4) If the jth index value of network sentiment emergency are fuzzy numbers $y_{i}=\left(y_{j 1}, y_{j 2}, y_{j 3}, y_{j 4}\right), x_{j}=\left(x_{j 1}, x_{j 2}\right.$,

$\left.x_{j 3}, x_{j 4}\right)$, then the distance [21] between them is

$$
d\left(y_{j}, x_{j}\right)=1-\frac{1}{1+\left|p\left(y_{j}\right)-p\left(x_{j}\right)\right|}
$$

where $p\left(y_{j}\right)=\frac{y_{j 1}+2 y_{j 2}+2 y_{j 3}+y_{j 4}}{6}$;

$$
p\left(x_{j}\right)=\frac{x_{j 1}+2 x_{j 2}+2 x_{j 3}+x_{j 4}}{6} .
$$

If the different importance of different warning decision index of network sentiment emergency should be taken into account, we can compute the following weighted distance of current emergency to source case as below. $d(y, x)=\sqrt{\sum_{j=1}^{n} w_{j} d^{2}\left(y_{j}, x_{j}\right)}$

where $w_{j}$ is the weight of the jth warning decision index of network public sentiment emergency.

Notably, the smaller of the weighted distance measure

value $d(y, x)$, the nearer of the source case $x$ to the unexpected network public sentiment emergency $y$.

\section{UNCERTAIN NETWORK PUBLIC SENTIMENT EMERGENCY DECISION MODEL BASED ON GREY RELATIONAL DEGREE}

As we know, grey relational theory [16] is an important analysis method in grey system, which can measure and compare the similarity and divergence between the current uncertain problem and old case problem. And grey related analysis reflects a form of fuzzification of input by using simple min-max operators, which is more suitable for dealing with MADM problem especially when sample size is small and sample attribute index is incomplete and unknown.

So, in the design of uncertain network sentiment emergency warning decision system we will adopt grey relation degree rather than similarity to measure the relational degree between any two network sentiment emergencies case and then find the most matching source case and rank all the priority order of candidate source cases in this warning decision developing system.

For sake of designing the uncertain network public sentiment emergency warning decision system, first we need to extract some significant warning decision index characteristics and identify the important warning decision index of uncertain network public sentiment emergency. And by using fuzzy AHP or other expert assignment methods, we can determine the weight of each network public opinion emergency index. Then by computing similarity or distance measure between significant warning decision indexes of the current unknown network sentiment emergency and some historical network sentiment emergencies we can also find a set of relatively matching candidate emergency case set and store them in the case base of the warning decision systems.

\subsection{State Description of Uncertain Network Public Sentiment Emergency Case}

In the case-based reasoning process of network sentiment emergency early warning decision system, the emergency knowledge and related expertise are expressed by data structure of case representation. The set of emergency case characteristics correspond to the set of warning decision index of network sentiment emergency. Let $X^{*}$ be the set of all the network sentiment emergency cases in the designed warning decision system. Each network sentiment emergency case can be regarded as a characteristic vector. Every dimension of this case characteristic vector corresponds to a warning decision index value of network sentiment emergency. And suppose $X \subseteq X^{*}$ is the possible matching $m$ characteristic 
vectors of the current unexpected emergency by using SQL search sentence under characteristic similar condition. If we identify $n$ significant warning decision index for uncertain network sentiment emergency system, then each of the characteristic vectors denotes a network sentiment emergency case with $n$ warning decision indexes.

Thus, the set of all the alternative network sentiment emergency cases can be represented by the following decision matrix.

$X=\left[\begin{array}{l}x_{1} \\ x_{2} \\ \cdots \\ x_{m}\end{array}\right]=\left[\begin{array}{ccccc}x_{11} & x_{12} & x_{13} & \cdots & x_{1 n} \\ x_{21} & x_{22} & x_{23} & \cdots & x_{2 n} \\ \cdots \ldots \ldots \ldots \ldots \ldots \ldots \ldots \ldots \ldots \ldots & \ldots \ldots \ldots \ldots \ldots \ldots \\ x_{m 1} & x_{m 2} & x_{13} & \cdots & x_{m n}\end{array}\right]$,

where each network public sentiment emergency case $x_{i}$ is represented by a row characteristic vector $\left(x_{i 1}, x_{i 2}, \ldots x_{i j}, \ldots, x_{i n}\right), x_{i j}$ represents the value of network sentiment emergency $x_{i}$ with respect to $j$ th warning decision index $c_{j}$, for $1 \leq j \leq n, 1 \leq i \leq m$.

And assume a new occurred unknown network public sentiment emergency is characterized by the following vector:

$y=\left(y_{1}, y_{2}, \cdots, y_{n}\right)$, where $y_{j}(1 \leq j \leq n)$ may be clear value and fuzzy value of the network public sentiment emergency index.

To facilitate government make efficient warning decision for this unexpected network public sentiment emergency, we should utilize the case reasoning mechanism of network public sentiment emergency warning decision system and retrieve the most desirable historical source case. So, we should also take the following procedures.

\subsection{Selection of the Most Matched Network Public Opinion Emergency Case}

From the resolution of historical emergency case and the collected index value of the unexpected network sentiment emergency, we can get a proper warning evaluation result and make efficient decision for the unknown network sentiment emergency. Thus, we need to calculate the similarity or distance measure between the new emergency and all the possible network sentiment emergencies, then finally select the most optimal source case to direct warning decision of each network sentiment emergency when the source case has the greatest similarity degree to the current unknown network sentiment emergency.

Step 1: In order to eliminate the effect of dimension, firstly we need to standardize the emergency case characteristic matrix into a normalized matrix

$$
X^{\prime}=\left(x_{i j}^{\prime}\right)_{m \times n} .
$$

The normalization processes of the network public sentiment emergency index are as follows:
If index $c_{j}$ is clear index, then the value of index $c_{j}$ can be normalized as below.

(1) when $c_{j}$ is a benefit index, the index value is normalized as

$x_{i j}^{\prime}=x_{i j} / \max _{1 \leq i s m} x_{i j}$

(2) when $c_{j}$ is cost index, the network public sentiment emergency index value is normalized as

$x_{i j}^{\prime}=\min _{1 \leq i \leq m} x_{i j} / x_{i j}$

If $c_{j}$ is interval valued index, then the value of index $c_{j}$ is normalized below.

(1) when $c_{j}$ is benefit index, the index value $x_{i j}=\left[x_{i j}^{-}, x_{i j}^{+}\right]$can be normalized as $x_{i j}^{\prime}=\left[x_{i j}^{\prime-}, x_{i j}^{\prime+}\right]$ by the following formulae.

$$
\begin{aligned}
x_{i j}^{\prime-} & =\left\{x_{i j}^{-}-\min _{i}\left(x_{i j}^{-}\right)\right\} /\left\{\max _{i}\left(x_{i j}^{+}\right)-\min _{i}\left(x_{i j}^{-}\right)\right\} \\
& x_{i j}^{\prime+}=\left\{x_{i j}^{+}-\min _{i}\left(x_{i j}^{-}\right)\right\} /\left\{\max _{i}\left(x_{i j}^{+}\right)-\min _{i}\left(x_{i j}^{-}\right)\right\} .
\end{aligned}
$$

(2) when $c_{j}$ is cost index, the interval index value is normalized as follows

$$
\begin{aligned}
& x_{i j}^{\prime-}=\left\{\max _{i}\left(x_{i j}^{+}\right)-x_{i j}^{+}\right\} /\left\{\max _{i}\left(x_{i j}^{+}\right)-\min _{i}\left(x_{i j}^{-}\right)\right\} \\
& x_{i j}^{\prime-}=\left\{\max _{i}\left(x_{i j}^{+}\right)-x_{i j}^{-}\right\} /\left\{\max _{i}\left(x_{i j}^{+}\right)-\min _{i}\left(x_{i j}^{-}\right)\right\} .
\end{aligned}
$$

Obviously, $0 \leq x_{i j}^{\prime-} \leq x_{i j}^{\prime+} \leq 1$.

In fact, from the above emergency decision matrix, one can see that a network sentiment emergency case is determined by $n$ early warning decision indexes. And every network sentiment emergency corresponds to a multidimensional point in $\mathrm{n}$ dimensional space. Therefore, matching and retrieving the most similar source case is analogue to find the closest point near to the current emergency object in $\mathrm{n}$ dimensional space.

In the subsequent, we shall adopt the grey relational theory to calculate the closeness of current network sentiment emergency with each source case in the designed network sentiment emergency decision system database.

Step 2: We compute the local grey relational degree of current network public sentiment emergency $y$ and historical source case $x_{i}(1 \leq i \leq m)$ regarding each warning decision index $c_{k}(1 \leq k \leq n)$ as follow:

$$
G\left(y_{j}^{\prime}, x_{i j}^{\prime}\right)=\frac{\min _{1 \leq i \leq m} \min _{1 \leq j \leq n} d\left(y_{j}^{\prime}, x_{i j}^{\prime}\right)+\lambda \max _{1 \leq i \leq m} \max _{1 \leq j \leq n} d\left(y_{j}^{\prime}, x_{i j}^{\prime}\right)}{d\left(y_{j}^{\prime}, x_{i j}^{\prime}\right)+\lambda \max _{1 \leq i \leq m} \max _{1 \leq j \leq n} d\left(y_{j}^{\prime}, x_{i j}^{\prime}\right)}
$$

where $\lambda \in[0,1]$ is discriminate coefficient, we generally take $\lambda=0.5$ for convenience.

Notably, the greater the value $G\left(y_{k}^{\prime}, x_{i k}^{\prime}\right)$ is, the more similar of current unexpected network sentiment emergency 
$y$ to historical network public sentiment emergency case $x_{i}$ $(1 \leq i \leq m)$ with respect to decision index $c_{j}(1 \leq j \leq n)$.

Step 3: We construct the following grey relational matrix.

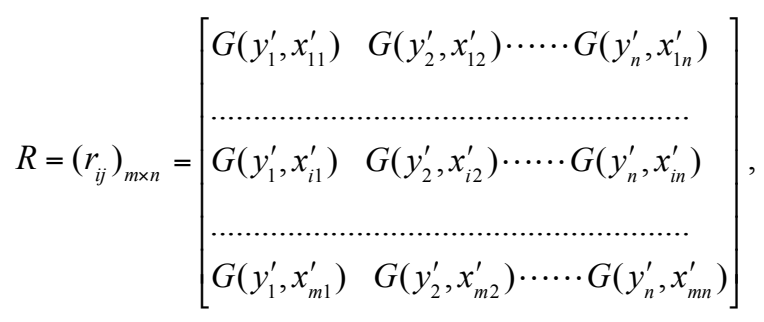

where $r_{i j}=G\left(y_{j}^{\prime}, x_{i j}^{\prime}\right)$ denotes the local grey relational degree between the new occurred unknown network public sentiment emergency and the $i$ th source case $x_{i}$ regarding $j$ th warning decision index $c_{j}$.

Since each warning decision index of network public opinion emergency has different importance, we generally consider the following comprehensive grey relational degree.

$r_{i}=G\left(y^{\prime}, x_{i}^{\prime}\right)=\sum_{j=1}^{n} w_{j} r_{i j}=\sum_{j=1}^{n} w_{j} G\left(y(j), x_{i}(j)\right)$

In fact, $r_{i}$ can measure the comprehensive grey relational degree between the current unexpected network public sentiment emergency $y$ and source case $x_{i}$ over all the warning decision indexes. The greater the value of $r_{i}$, the more similarity between the current network public sentiment emergency $y$ and historical source emergency case $x_{i}$.

Step 4: Rank the comprehensive grey relational degree of current emergency with each source case in this designed network sentiment emergency warning decision system.

If $r_{i}<r_{s}$, then the similarity between current emergency $y$ and source case $x_{i}$ is smaller than that between current emergency and source case $x_{s}$. That is to say, the current unknown network public sentiment emergency is closer to source case $x_{s}$ than $x_{i}$.

If $t=\arg \max \left\{r_{i} / 1 \leq i \leq m\right\}$, then the most matching case of unknown network sentiment emergency is source case $x_{t}$. Thus, we can use the early warning decision resolution of historical network sentiment emergency case $x_{t}$ to facilitate the quick warning decision for the unexpected network public sentiment emergency.

\section{NUMERICAL EXAMPLE}

Recently, grey relational theory is widely used in uncertain decision system. In this section, we will give one numeric example to demonstrate the application of the proposed comprehensive grey relational degree to case-based reasoning model of uncertain network sentiment emergency warning decision system involved with many uncertain indexes.

Example 1. Suppose there are four historical network sentiment emergency source cases stored in the designed network sentiment emergency warning decision system. And the important warning decision index values of these stored source cases are recorded in Table 1. Once a new unexpected network sentiment emergency occurs in some area, we need to forecast the warning degree and determine the proper warning decision resolution from the existing historical network public sentiment emergency case data to facilitate urgent decision for the current unknown emergency. Assume the uncertain index values of this unknown network sentiment emergency are also collected and displayed in Table $\mathbf{1 .}$ By fuzzy AHP method, assume we select the following eight important warning decision indexes of the network sentiment emergency, and get the weight vector $(0.05,0.1,0.15,0.2$, $0.1,0.05,0.2,0.15)$ of all the significant warning decision indexes. And in the case base of designed network sentiment emergency early warning decision system, suppose the maximum blog number is 10,0000 , maximum browser number is 300,0000 , maximum reply mumber is 2,00000 for all network public opinion emergencies in the designed emergency system. As stated in literature [22], the uncertain linguistic values in the emergency decision system Table 2 correspond to the fuzzy assessment numbers as in Table $\mathbf{1 .}$

Firstly, by using the above formulae (6), (8) we can transfer all the fuzzy linguistic value in the above early warning decision system Table $\mathbf{1}$ to the corresponding fuzzy numbers listed in the following normalized warning decision system Table $\mathbf{3}$ for uncertain network public sentiment emergency.

Secondly, from the proposed different distance measure formulae (1)-(5) in section 2 we can compute the following distances between the index values of the current network sentiment emergency and each historical emergency case stored in the case-base of the uncertain network public sentiment emergency warning decision system as below.

$$
\begin{aligned}
& d\left(y_{1}, x_{11}\right)=0.3888, d\left(y_{2}, x_{12}\right)=0.4652, \\
& d\left(y_{3}, x_{13}\right)=0.1496, d\left(y_{4}, x_{14}\right)=0.8571 \\
& d\left(y_{5}, x_{15}\right)=0.3, d\left(y_{6}, x_{16}\right)=0.4231 \\
& d\left(y_{7}, x_{17}\right)=0.75, d\left(y_{8}, x_{18}\right)=0.75 \\
& d\left(y_{1}, x_{21}\right)=0.007, d\left(y_{2}, x_{22}\right)=0.1367 \\
& d\left(y_{3}, x_{23}\right)=0.1005, d\left(y_{4}, x_{24}\right)=0.5714 \\
& d\left(y_{5}, x_{25}\right)=0.1, d\left(y_{6}, x_{26}\right)=0.1111 \\
& d\left(y_{7}, x_{27}\right)=0.5714, d\left(y_{8}, x_{28}\right)=0.6 ; \\
& d\left(y_{1}, x_{31}\right)=0.0756, d\left(y_{2}, x_{32}\right)=0.2118 \\
& d\left(y_{3}, x_{33}\right)=0.3111, d\left(y_{4}, x_{34}\right)=0.6
\end{aligned}
$$




$$
\begin{aligned}
& d\left(y_{5}, x_{3}\right)=0.1, \quad d\left(y_{6}, x_{36}\right)=0.0786, \quad d\left(y_{7}, x_{37}\right)=0.6, \\
& d\left(y_{8}, x_{38}\right)=0.6 ; \\
& d\left(y_{1}, x_{41}\right)=0.1546, d\left(y_{2}, x_{42}\right)=0.0409 \\
& d\left(y_{3}, x_{43}\right)=0.2029, d\left(y_{4}, x_{44}\right)=0.75 \\
& d\left(y_{5}, x_{45}\right)=0.2, d\left(y_{6}, x_{46}\right)=0.3514 \\
& d\left(y_{7}, x_{47}\right)=0.8182, d\left(y_{8}, x_{48}\right)=0.7391 .
\end{aligned}
$$

Again from formula (10), we obtain the following grey relational degree matrix.

$R=\left(r_{i j}\right)_{4 \times 8}=$

$\left[\begin{array}{llllllll}0.5329 & 0.4873 & 0.7534 & 0.3388 & 0.5978 & 0.5114 & 0.3696 & 0.3696 \\ 1.0000 & 0.7705 & 0.8233 & 0.4356 & 0.8240 & 0.80710 & 0.4356 & 0.4235 \\ 0.8639 & 0.6802 & 0.5889 & 0.4235 & 0.8240 & 0.85880 & 0.4235 & 0.4235 \\ 0.7469 & 0.9278 & 0.6898 & 0.3696 & 0.5978 & 0.55840 & 0.3493 & 0.3730\end{array}\right]$.

According to weighted grey relational coefficient formula (11), we get the comprehensive grey relational degree of each historical source case to the current unknown network public sentiment emergency.

$$
\begin{aligned}
& r_{1}=\sum_{j=1}^{8} w_{j} r_{1 j}=0.4709 ; r_{2}=\sum_{j=1}^{8} w_{j} r_{2 j}=0.6111 ; \\
& r_{3}=\sum_{j=1}^{8} w_{j} r_{3 j}=0.5578 ; r_{4}=\sum_{j=1}^{8} w_{j} r_{4 j}=0.5305 .
\end{aligned}
$$

Since $\quad r_{2}=\max \left\{r_{i} / 1 \leq i \leq 4\right\}, \quad$ i.e., $\quad 2=\operatorname{argmax}$ $\left\{r_{i} / 1 \leq i \leq 4\right\}$, we can see that historical case 2 has the greatest relational degree with the current network sentiment emergency problem. Therefore, the historical network sentiment emergency Case 2 is the nearest one to the current unknown network sentiment emergency That is to say, Case 2 is the most desirable retrieval source case in all the candidate matching emergencies in this designed warning decision system. The resolution of historical case $x_{2}$ is the optimal resolution of the new occurred network public sentiment emergency $y$. Thus, the related emergency management department can employ or modify the warning decision resolution of the matching network sentiment emergency case 2 based on grey relation retrieval algorithm to facilitate the urgent decision for the current unknown network sentiment emergency. And the related emergency section can also implement the corresponding emergency response to cope with this network sentiment emergency and raise the corresponding early warning and take urgent decision mechanism to coordinate all kinds of emergency facilities among different municipal zones to avoid or decrease the risk loss of the unexpected network public sentiment emergency.

Table 1. Fuzzy number assessment for fuzzy linguistic values.

\begin{tabular}{|c|c|}
\hline Fuzzy Linguistic Values & Fuzzy Numbers \\
\hline \hline Extremely quick / Extremely serious & $(8,9,10,10)$ \\
\hline Very quick / Very serious & $(7,8,8,9)$ \\
\hline Quick/ Serious & $(5,6,7,8)$ \\
\hline Medium & $(4,5,5,6)$ \\
\hline Slow / Slight & $(2,3,4,5)$ \\
\hline Very Slow / Very slight & $(1,2,2,3)$ \\
\hline Extremely slow / Extremely slight & $(0,0,1,2)$ \\
\hline
\end{tabular}

\begin{tabular}{|c|c|c|c|c|c|}
\hline & Case 1 & Case 2 & Case 3 & Case 4 & Case y \\
\hline Blog & 11247 & 49426 & 87691 & 45590 & 50128 \\
\hline Browser & 624208 & 2429889 & 1384470 & 1142575 & 2019780 \\
\hline Replier & 7720 & 12722 & 16933 & 6654 & 10712 \\
\hline Sentiment spread speed & slow & Extremely quick & quick & Medium & Very quick \\
\hline Public Sentiment tendency & $<0.5,0.4>$ & $<0.9,0.1>$ & $<0.7,0.2>$ & $<0.6,0.3>$ & $<0.8,0.1>$ \\
\hline Economic loss & {$[100,300]$} & {$[200,900]$} & {$\left[\begin{array}{ll}400 & 800\end{array}\right]$} & {$\left[\begin{array}{ll}700 & 1000\end{array}\right]$} & {$[300,800]$} \\
\hline Alarm severity & medium & Extremely serious & serious & $(2,3,4,5)$ & very serious \\
\hline Response speed & slow & Very quick & medium & Extremely quick & quick \\
\hline
\end{tabular}

\section{CONCLUSION}

Several authors have studied the warning decision models of network public opinion emergency. Those models are mainly based on the distance and similarity between the current public sentiment emergency problem and the historical case. In the present study, we use the grey relational degree

Table 2. Uncertain network public sentiment emergency warning decision system. 
Table 3. Normalized decision system for uncertain network public sentiment emergency.

\begin{tabular}{|c|c|c|c|c|c|c|c|c|}
\hline & Blog & Browser & Replier & $\begin{array}{c}\text { Sentiment } \\
\text { Spread Speed }\end{array}$ & $\begin{array}{l}\text { Sentiment } \\
\text { Tendency }\end{array}$ & $\begin{array}{l}\text { Economic } \\
\text { Loss }\end{array}$ & $\begin{array}{c}\text { Alarm } \\
\text { Severity }\end{array}$ & $\begin{array}{c}\text { Response } \\
\text { Speed }\end{array}$ \\
\hline Case $x 1$ & 0.1125 & 0.2081 & 0.386 & $(1,2,2,3)$ & $<0.5,0.4>$ & {$[0,0.2222]$} & $(4,5,5,6)$ & $(2,3,4,5)$ \\
\hline Case $x 2$ & 0.4943 & 0.81 & 0.6361 & $(8,9,10,10)$ & $<0.9,0.1>$ & $\begin{array}{l}{[0.1111} \\
0.8889]\end{array}$ & $(8,9,10,10)$ & $(7,8,8,9)$ \\
\hline Case $x 3$ & 0.5769 & 0.4615 & 0.8467 & $(5,6,7,8)$ & $<0.7,0.2>$ & $\begin{array}{c}{[0.3333,} \\
0.7778]\end{array}$ & $(5,6,7,8)$ & $(4,5,5,6)$ \\
\hline Case $x 4$ & 0.6559 & 0.7142 & 0.3327 & $(4,5,5,6)$ & $<0.6,0.3>$ & {$[0.6667,1]$} & $(2,3,4,5)$ & $(8,9,10,10)$ \\
\hline Case $Y$ & 0.5013 & 0.6733 & 0.5356 & $(7,8,8,9)$ & $<0.8,0.1>$ & $\begin{array}{c}{[0.2222} \\
0.7778]\end{array}$ & $(7,8,8,9)$ & $(5,6,7,8)$ \\
\hline
\end{tabular}

to measure the matching degree between the unexpected network sentiment emergency and source cases in uncertain warning decision system, which gives an idea of the improvement of the quality of warning degree prediction and emergency decision case matching by taking into account the weight of all the warning decision indexes of uncertain network public opinion emergency.

\section{CONFLICT OF INTEREST}

The authors confirm that this article content has no conflict of interest.

\section{ACKNOWLEDGEMENTS}

This work is supported by the National Social Science Foundation of China (13CGL130), the Natural Science Foundation of Guangdong Province (S2013010013050, S2012040007184), the Scientific and Technological Innovation Project of Guangdong Province Colleges Subject Construction Special Fund (2013KJCX0069), the National Natural Science Foundation (61202271), the Humanities and Social Sciences Research Youth Foundation of Ministry of Education of China (13YJCZH258), and the Innovative School Project in Higher Education of Guangdong, China (GWTP-GC-2014-03)- High Level Talents Project in Guangdong Province.

\section{REFERENCES}

[1] Z. H. Hu, "Multi-signal cooperative decision for emergency management inspired by immune system," Journal of Computers, vol. 5, no. 9, pp. 1410-1416, 2010.

[2] Y. J. Peng, Z.B. Hu and X. Guo, "Research on the evolution law and response capability based on resource allocation model of unconventional emergency," Journal of Computers, vol. 5, no. 12, pp. 1899-1906, 2010

[3] K. J. Liao, H. H. Xiong, D. H. Ye and C.Y. Zhang, "A method of emergency management based on knowledge element theory," Journal of Software, vol. 7, no. 1, pp. 41-48, 2012.

[4] R. X. Zeng, "Construction of network public sentiment emergency early warning index system," Information Studies: Theory and Application, vol. 33, no. 1, pp. 77-80, 2010.

[5] Y. W. Zhang, J. Y. Qi, B.X. Fang, and Y. X. Li, “ Research on the index system of public opinion on internet for unexpected emer- gency, " Journal of Information Science, vol. 29, no. 9, pp. 14181424, 2011.

[6] B. Shi, "The decision mechanism and strategy for public emergency," Information Studies-Theory and Application, vol. 33, no. 7, pp. 93-96, 2010.

[7] R. X. Zen and X. L. Xu, "A study on early warning mechanism and index of network public opinion emergency," Journal of Intelligence, vol. 28, no. 11, pp. 52-54, 2009.

[8] C. Lin, B. C. Li and J. Wang, "Network public sentiment early warning method based on fuzzy reasoning," Journal of Information Engineering University, vol. 12, no. 1, pp. 72-76, 2011.

[9] Y. Dai, X.W. Hao, Y. Guo and Z.H. Yu, "Safe evaluation model for network public sentiment based on multi-level fuzzy comprehensive evaluation," Network Information Safety, vol. 5, pp. 60-62, 2010.

[10] Y. T. Li, Y. Liu and Y. Liu, "Study on the grey early warning evaluation of network public sentiment," Journal of Intelligence, vol. 30 , no. 4, pp. $24-27,2011$

[11] K.F. Xie, "The life cycle principle and group decision for network public emergency," Journal of Wuhan University of Technology, vol. 23, no. 4, pp. 482-486, 2010.

[12] Y. F. Zhang and C. He, "Intelligent analysis for network public sentiment based on web mining," Information Studies: Theory and Application, vol. 34, no. 4, pp. 64-67, 2011.

[13] Peking University Fangzheng intelligent public sentiment aided decision support system, http: //www.founderrd.com/2006-03/16.

[14] Goonie network public sentiment supervision analysis system, http://www.goonie.cn/products//2008/01/content3.html

[15] Q.Y. Zhong, S. Guo, X. Ye, and Y.G. Zhang, "Case representation and retrieving method for emergency aided decision," Journal of DaLian University of Technology, vol. 51, no. 1, pp. 137-142, 2011

[16] Z. W. Liao, "Financial disasters pre-warning based on CBR and gray correlation degree," Computer Engineering, vol. 38, no. 1, pp. 174-179, 2012.

[17] X. L. Liu, B. J. Tang, and W. H. Qiu, "A search model for enterprise crisis early-warning cases based on gray association," China Soft Science, vol. 22, no. 8, pp. 152-160, 2007.

[18] D. Y. Chang, "Applications of the extent analysis method on fuzzy AHP," European Journal of Operational Research, vol. 95, no. 3, pp. 649-655, 1996.

[19] F. E. Boran, S. Genc, M. Kurt and D. Akay, "A multi-criteria intuitionistic fuzzy group decision making for supplier selection with Toposis method," Expert Systems with Applications, vol. 36, pp. 11363-11368, 2009.

[20] S. W. Shi, H.L. Huang and M.L. Lin, "New distance measure of intuitionistic fuzzy sets," International Journal of Applied Mathematics and Statistics, vol. 49, no. 19, pp. 6-25, 2013. 
[21] C. H. Hsieh and S. H. Chen, "Similarity of generalized fuzzy numbers with graded mean integration representation," In: Proceedings of the 8th International Fuzzy Systems Association World Congress, vol. 2, pp. 551-555, 1999.
[22] C.C. Chou, F.T. Kuo, R. H. Gou, C.L. Tsai, C.P. Wong, and M.C. Tsou, "Application of a combined fuzzy multiple criteria decision making and optimization programming model to the container transportation demand split", Applied Soft Computing, vol. 10, pp. 1080-1086, 2010.

Received: September 22, 2014

Revised: November 30, 2014

Accepted: December 02, 2014

(C) Zhang et al.; Licensee Bentham Open.

This is an open access article licensed under the terms of the Creative Commons Attribution Non-Commercial License (http://creativecommons.org/licenses/by-nc/3.0/) which permits unrestricted, non-commercial use, distribution and reproduction in any medium, provided the work is properly cited. 